\title{
Atuação interprofissional vivenciada no Programa de Educação pelo Trabalho pela Saúde: um relato de experiência
}

\author{
Interprofessional performance experienced in the Education through Work for Health \\ Program: an experience report
}

\section{Desempeño interprofesional experimentado en el Programa de Educación para el Trabajo en Salud: un relato de experiencia}

Matheus Albino Souza ${ }^{1 *}$, Ricardo Zanella ${ }^{1}$, Carolina Geraldi da Silva ${ }^{1}$, Gabriele Nichetti Vanin ${ }^{1}$, Laura Zanella ${ }^{1}$, Natália Weber do Amaral ${ }^{1}$, Nathália Longo Nunes ${ }^{1}$ e Raquel Dalcim¹.

\section{RESUMO}

Objetivo: Expor as atividades realizadas a partir da reflexão da interprofissionalidade e dos novos paradigmas do cuidado em saúde, evidenciando assim, a qualificação acadêmico-profissional que acontece com iniciativas como essa. Relato de experiência: $O$ presente trabalho relata as experiências vivenciadas entre os anos de 2019 e 2021 por um grupo de bolsistas do Programa de Educação pelo Trabalho para a Saúde (PET-Saúde), grupo este composto por acadêmicos de diferentes áreas da saúde, que ao longo de dois anos atuaram em uma Unidade Básica de Saúde (UBS) realizando atividades interprofissionais que proporcionavam uma ligação entre ensino-serviço-comunidade. Dentre essas atividades estavam: acompanhamento de consultas, visitas domiciliares, participação em grupos de apoio, entre outras. Considerações finais: O programa PET-Saúde proporcionou aos participantes uma série de aprendizados e experiências que envolveram o trabalho interprofissional e aproximaram o estudante da realidade da sua comunidade e do sistema de saúde brasileiro. Contribuindo com a formação destes futuros profissionais, que saíram do programa mais preparados para o trabalho em equipe e para realizar um cuidado em saúde integral.

Palavras-chave: Educação interprofissional, Educação em saúde, Relações comunidade-instituição.

\begin{abstract}
Objective: To expose the activities carried out from the reflection of the interprofessionality and the new paradigms of health care, thus showing the academic-professional qualification that happens with initiatives like this. Experience report: The present work reports the experiences lived between the years 2019 and 2021 by a group of scholarship holders from the Education through Work for Health Program (PET-Saúde), a group composed of academics from different areas of health, who over two years worked in a Basic Health Unit (UBS) performing interprofessional activities that provided a link between teaching-service-community. Among these activities were: follow-up consultations, home visits, participation in support groups, among others. Final considerations: The PET-Saúde provided participants with a series of learnings and experiences that involved interprofessional work and brought students closer to the reality of their community and the Brazilian health system. Contributing to the training of these future professionals, who left the program more prepared for teamwork and to provide comprehensive health care.
\end{abstract}

Keywords: Interprofessional education, Health education, Community-institutional relations.

1 Universidade de Passo Fundo (UPF), Passo Fundo - RS. *E-mail: matheussouza@upf.br 


\section{RESUMEN}

Objetivo: Exponer las actividades realizadas en base a la reflexión de la interprofesionalidad y los nuevos paradigmas del cuidado de la salud, mostrando así la calificación académico-profesional que se da con iniciativas como esta. Informe de experiencia: El presente trabajo relata las experiencias vividas entre los años 2019 y 2021 por un grupo de becarios del Programa Educación por el Trabajo para la Salud (PETSaúde), grupo integrado por académicos de diferentes áreas de la salud, que durante dos años trabajaron en una Unidad Básica de Salud (UBS) realizando actividades interprofesionales que proporcionaban un vínculo entre docencia-servicio-comunidad. Entre estas actividades se encuentran: consultas de seguimiento, visitas domiciliarias, participación en grupos de apoyo, entre otras. Consideraciones finales: EI PET-Saúde brindó a los participantes una serie de aprendizajes y experiencias que involucraron el trabajo interprofesional y acercaron a los estudiantes a la realidad de su comunidad y del sistema de salud brasileño. Contribuyendo a la formación de estos futuros profesionales, que salieron del programa más preparados para el trabajo en equipo y para brindar una atención integral de la salud.

Palabras clave: Educación interprofesional, Educación en salud, Relaciones comunidad-institución.

\section{INTRODUÇÃO}

O antigo modelo de cuidado em saúde, onde cada profissional trabalha sozinho para resolver a uma demanda específica do paciente, vem sendo contestado, e diante disso, no mundo todo, houve um crescimento das práticas interprofissionais. A interprofissionalidade contrapõe-se às relações tradicionais hierarquizadas, pois envolve um constante processo de comunicação e de tomada de decisões conjuntas, que permite que os conhecimentos e habilidades de diferentes profissionais atuem de forma sinérgica com o usuário e a comunidade, proporcionando um cuidado em saúde mais abrangente (MATUDA CG, et al., 2015).

O percurso do Sistema Único de Saúde brasileiro se caracterizou por importantes mudanças na atenção à saúde da população, como mostra Viacava $\mathrm{F}$, et al. (2018), porém, para que este modelo de trabalho seja implantado no Sistema Único de Saúde (SUS) é preciso primeiramente repensar a formação dos profissionais. Pois, segundo Batista NA (2012), até pouco tempo, cada aluno que adentrava um curso de saúde aprendia e convivia somente com seus pares e jamais com profissionais de outras áreas, o que acabava por reforçar a problemática da fragmentação do cuidado.

Dessa forma, nas últimas décadas, o Ministério da Saúde e o da Educação uniram-se para reavaliar a educação formal dos profissionais de saúde no Brasil, com o objetivo de integralizar o cuidado e torna-lo interprofissional. Para tal, as Diretrizes Curriculares Nacionais (DCN) e o SUS foram utilizados como norteadores, conforme Farias-Santos BC e Noro LR (2017), para garantir uma formação voltada para as práticas de atenção, trabalho e construção do conhecimento a partir das necessidades reais da população. Essa parceria gerou um aumento de políticas de integração entre as instituições de ensino e os serviços da saúde, sendo o Programa de Educação pelo Trabalho para Saúde (PET-Saúde) um de seus principais frutos.

O PET-Saúde surgiu no ano de 2009 para subsidiar a formação de profissionais da saúde e para atender a perfis socioepidemiológicos da população, e tem como objetivo a integração ensino-serviço-comunidade. Para isso, o programa utiliza estratégias que privilegiam a prática na rede de saúde como protagonista no processo de ensino e aprendizagem dos participantes. Haddad AE, et al. (2012), afirma que a educação acontece através do trabalho em uma parceria que envolve o estudante, os docentes, a equipe de saúde e também seus respectivos usuários.

O programa tem duração de dois anos e é composto por acadêmicos universitários bolsistas ou voluntários que atuam diretamente na comunidade em Unidades de Saúde (UBS) e nas secretarias municipais de saúde; profissionais de saúde que são preceptores responsáveis por acompanhar o trabalho dos alunos na comunidade; docentes dos cursos que são tutores e supervisionam o trabalho dos alunos e participam também indiretamente do trabalho na comunidade; coordenação do projeto responsável pela organização 
geral e pela manutenção das atividades grupais. Dessa forma, a equipe do PET-Saúde atende aos usuários da rede, prezando pela integralidade e pela humanização do cuidado (SOUSA FM, et al., 2020).

Considerando que o cuidado em saúde é dinâmico e, por isso, mutável, uma reflexão por parte dos profissionais de saúde acerca das práticas que envolvem o seu saber/fazer do cotidiano de trabalho pode contribuir para que melhorias aconteçam no atendimento ao usuário. Conforme Sousa FM, et al. (2020), é através de uma avaliação do trabalho e dos conhecimentos obtidos até o momento, que se pode fortalecer aquilo que já é bom e dar atenção àquilo que precisa ser mais bem desenvolvido.

Assim, este trabalho tem por objetivo de relatar as atividades realizadas a partir da reflexão da interprofissionalidade e dos novos paradigmas em relação aos cuidados em saúde, focando na ideia de uma única saúde, evidenciando assim, a qualificação acadêmico-profissional que acontece com estas iniciativas.

\section{RELATO DE EXPERIÊNCIA}

Este relato baseia-se na experiência de bolsistas do PET-Saúde, desenvolvido pelo Ministério da Saúde em parceria com a Secretária Municipal de Saúde de Passo Fundo- RS e a Universidade de Passo Fundo (UPF). Sendo que o grupo aqui referido era composto por seis acadêmicas dos cursos de: Medicina, Odontologia, Medicina Veterinária, Nutrição, Psicologia e Fonoaudiologia.

O programa foi desenvolvido por um periodo de dois anos, tendo seu início em março de 2019 e seu término em março de 2021. Os participantes eram separados em quatro subgrupos compostos por: alunos, preceptores e professores de diversas áreas da saúde. Assim, cada equipe atuava em um bairro da cidade, sendo que o nosso grupo atuava na UBS do bairro Zacchia localizado na cidade de Passo Fundo -RS. Ao longo desses anos de projeto vivenciou-se muitas experiências, tendo conhecimento de realidades distintas e o desafio de adaptar o trabalho ao cenário pandêmico, e suas demandas tão distintas de tudo aquilo que foi experimentado até então.

No primeiro ano as atividades ficaram concentradas na UBS e dentre elas estavam o acompanhamento de consultas, o desenvolvimento de grupos e salas de espera, a confecção de materiais e as visitas domiciliares.

Foi acompanhado consultas realizadas pelos enfermeiros que eram preceptores e de acordo com os conhecimentos particulares de cada um, foi possível ajudar com a consulta. Posteriormente, organizavam junto aos alunos residentes diversos grupos como os de: gestantes, tabagismo e diabetes. Os grupos eram semanais e compostos por usuários da rede que possuíssem indicação e interesse em participar. $O$ trabalho em equipe era o que se sobressaia, pois era preciso planejar de antemão aquilo que seria trabalhado posteriormente em grupo, e ter uma boa aliança de trabalho para transmitir confiança ao participante.

Já nas atividades de sala de espera, eram preparados materiais para serem abordados brevemente com quem ali estivesse aguardando para ser atendido. Dentre os temas escolhidos estavam dicas para dormir melhor, alcoolismo, dicas de alimentação saudável e zoonoses. Alguns desses temas foram escolhidos pelos alunos e outros foram sugeridos pelos usuários, que deixavam suas sugestões em uma caixa localizada na entrada da UBS. Assim, durante a espera, era falado para a comunidade desses temas de maneira rápida e acessível, buscando instruí-los e aproximá-los da unidade, ao mesmo tempo em que os alunos aprendiam sobre diversas áreas da saúde.

Além disso, foram confeccionados materiais educativos para exposição, cartazes com conteúdo como prevenção de doenças mentais, zoonóticas, dicas para insônia etc. Participou-se também na confecção de etiquetas para medicamentos que continham a quantia e a frequência de uso adequada, para facilitar 0 entendimento dos pacientes, o que contribuiu significativamente com o conhecimento sobre medicamentos em geral, já que as alunas em questão eram totalmente leigas na área farmacêutica.

Toda semana, participou-se das reuniões de equipe, onde tiveram a oportunidade de visualizar as dificuldades de uma equipe interprofissional, além de poder dar ideias e opiniões, enriquecendo o crescimento da equipe. Ademais, essa inserção nas reuniões foi de grande valor para que o grupo fosse reconhecido como parte da equipe Zacchia e não apenas como visitantes. 
As visitas domiciliares e os mapeamentos possibilitaram uma exploração do território da cidade, e consequentemente, uma maior aproximação do estudante com a comunidade. Estas eram realizadas juntamente com os residentes, enfermeiros e agentes comunitários da unidade, sendo que os alunos de diferentes cursos acompanhavam e complementavam com o conhecimento adquirido, vivenciando na prática a interprofissionalidade.

Como um dos tutores e uma das alunas integrantes de nossa equipe eram da área da Medicina Veterinária participamos da confecção de formulários que buscaram saber a quantidade de animais não castrados no bairro e ajudar na organização de projetos municipais voltados ao cuidado animal e atuamos na resolução de situações importantes para o bem-estar de animais da comunidade. Ademais, desenvolveram-se atividades em escolas da comunidade, com o objetivo de levar conhecimento sobre saúde para crianças e adolescentes.

No segundo ano, em contexto da pandemia, o PET-Saúde precisava de mudanças estratégicas de intervenção para dar recursos à população em geral bem como aos trabalhadores da saúde. Diante dessa situação a equipe PET-SAÚDE se reorganizou estrategicamente nos projetos: Redes de Cuidados à População e Saúde do Trabalhador. O projeto Rede de Cuidados tinha como objetivo identificar a população de maior vulnerabilidade social e grupos de risco na comunidade, através de um questionário. No projeto da saúde do trabalhador, desenvolveu-se atividades online semanais voltadas para os trabalhadores da saúde, trabalhando temas de autocuidado físico e mental.

Além das experiências na UBS e nas ruas das comunidades, também realizou pesquisas acadêmicas. $O$ grupo pôde apresentar trabalhos acadêmicos e dividir seus conhecimentos em eventos como a Semana do Conhecimento da UPF, promovendo a divulgação do projeto.

Dessa forma, percebe-se que ao longo desses dois anos, ocorreu uma inserção real no serviço e na comunidade, de modo que essas vivências contribuíram para nossa formação enquanto futuros profissionais e enquanto cidadãs.

\section{DISCUSSÃO}

No decorrer dos anos, o dinamismo do cuidado em saúde foi revelando diferentes demandas para seu aprimoramento e para a formação acadêmica e profissional. Sendo que, diante dessa realidade, um dos maiores desafios encontrados pelas instituições de ensino é na formação, construção e operacionalização de currículos que possibilitem o trabalho interprofissional, conforme Cervo, EB et al. (2020). Pois a interprofissionalidade é capaz de melhorar os resultados do trabalho em saúde, já que contribui positivamente para um cuidado mais eficiente com o usuário e possibilita uma ampliação da percepção e compreensão entre a equipe. Em consequência disso, as relações de trabalho tornam-se mais favoráveis (PREVIATO G e BALDISSERA V, 2018).

De acordo com Peduzzi, M (2016), um dos principais desafios para a implantação da interprofissionalidade está na articulação entre essas atividades e as atividades específicas de cada área profissional, visto que quando unidas estas permitem o aprendizado conjunto de competências necessárias para o trabalho em equipe e a prática interprofissional. Segundo Machado, MH e Neto, FR (2018), a gestão do trabalho vinculado com a educação na atuação nas áreas da saúde tem chamado a atenção dos gestores e de instituições que buscam uma adequação entre as necessidades da população associados aos objetivos institucionais. No que tange o tema principal do programa, a interprofissionalidade foi vivida na prática em todos os dias enquanto bolsista, pois convivíamos quase que exclusivamente com profissionais de outras áreas, e assim aprendeuse um pouco mais sobre as áreas da saúde como um todo.

Nesse sentido, o maior desafio do projeto foi reconhecer até onde é possivel adentrar o saber do outro, e até que ponto este deveria ser de sua exclusividade. Pois, é claro que mesmo em uma equipe interprofissional, um estudante de psicologia, por exemplo, não terá as mesmas habilidades de um estudante de odontologia, e algumas técnicas mais específicas e particulares de cada área permanecem sendo exclusivas àquele profissional. Por outro lado, quando se fala em cuidado integrativo, voltado principalmente à prevenção, há pouco que não pode ser feito de maneira interprofissional (CECCIM RB, 2018). 
Assim, com o trabalho em equipe, foi possível somar conhecimentos e aprender coisas novas que jamais aprenderíamos ao longo da graduação tradicional. A estudante de psicologia aprendeu como funciona uma farmácia, a estudante de nutrição aprendeu com a estudante de fonoaudiologia, que aprendeu com a de medicina, que aprendeu com a de medicina veterinária. O que corrobora com os resultados encontrados por Grzybowski LS, et al. (2017), no qual a maioria dos bolsistas ao sair do projeto afirmaram que aprenderam novas possibilidades de atuação profissional, constatando como é importante o aluno da área da saúde prender dentro do SUS, além da teoria de sala de aula.

Portanto, conforme Carvalho YM e Ceccim RB (2012) a graduação na área da saúde não tem tido uma orientação integradora entre ensino e trabalho, que esteja voltada para uma formação teórico-conceitual e metodológica que potencialize competências para a integralidade, onde se inclui o enfrentamento das necessidades de saúde da população e de desenvolvimento do sistema de saúde, sendo essencial discutir a formação em saúde no âmbito da graduação nas profissões dessa área, o PET-SAÚDE é um modelo de projeto em que os alunos da área da saúde estão inseridos nas unidades básicas, entendendo, diagnosticando e tratando os problemas de uma comunidade.

Dessa forma, os acadêmicos participantes do programa PET-SAÚDE, avaliaram o indivíduo com um olhar mais amplo, desde o momento de diagnosticar a doença até a conduta que devia ser tomada, pois as demandas dos usuários eram discutidas em reuniões de equipe compostas por diferentes áreas profissionais como descrito por (SILVA AN, et al. 2020). Com o PET-SAÚDE também foi possível se aproximar das crianças e adolescentes do bairro, foram feitas dinâmicas de ensino em saúde nesses locais, visto que, como cita Gueterres EC, et al. (2017) o contexto escolar é essencial para o desenvolvimento do conhecimento partilhado e para a integração com a comunidade.

Conforme Ribeiro KG, et al. (2018), as escolas possuem papel fundamental na criação de ambientes saudáveis, na perspectiva da promoção da saúde. Para Grando MK e Dall'agnol CM (2010), as reuniões de uma equipe são responsáveis por construir uma estrutura, capaz de chegar a melhor maneira de resolver as discussões, e é essencial o planejamento em conjunto para obter-se um melhor resultado nas decisões tomadas.

Segundo Morais FR, et al. (2012) o principal objetivo do PET-Saúde é a interação entre ensino, serviço e comunidade. Consideramos que esse objetivo foi atingido, pois foi possível conhecer o cotidiano da UBSZacchia, e posteriormente de outros bairros no Projeto Redes de Cuidado, além de conhecer o território passofundense, com suas demandas únicas. É muito importante notar que não só os estudantes puderam interagir com a comunidade, mas também as comunidades puderam conhecer aqueles que estão se preparando para atendê-los no futuro. Para Ferreira VS, et al. (2012) essa interação entre comunidade e estudantes ajuda a aproximar a serviço de saúde do usuário e consequentemente, os trabalhos realizados pela equipe são aceitos com mais facilidade.

As teorias e metodologias da Educação Interprofissional (EIP) em saúde tornaram-se globalmente reconhecidas como importantes ferramentas no desenvolvimento de competências colaborativas para que o trabalho em equipe possa ser realizado adequadamente Costa MV, et al. (2019). Ao término do programa, saímos todos profissionais da saúde mais completos e mais preparados para dividir seus conhecimentos e para aprender uns com os outros. As ligações feitas entre os cursos ali formadas, são vínculos que, mantidos, representam o início de uma mudança maior dentro da formação acadêmica em saúde.

Através desse relato de experiência foi possivel refletir sobre o impacto do PET-Saúde em nossa formação enquanto futuros profissionais de saúde. Com isso, o programa traz aos seus participantes aprendizados e experiências únicas, que um estudante de graduação dificilmente possui. Todos esses conhecimentos farão a diferença não somente no relacionamento entre a equipe de trabalho e em nossa qualificação profissional, mas também na qualidade do atendimento fornecido para o usuário. Dessa forma, busca com este relato possa motivar outros estudantes e profissionais a participar do PET-Saúde e também promova uma maior valorização do mesmo. 


\section{REFERÊNCIAS}

1. BATISTA NA. Educação Interprofissional em Saúde: concepções e práticas. FNEPAS, 2012; 2: 25-28.

2. CARVALHO YM, CECCIM RB. Formação e Educação em Saúde: aprendizados com a Saúde Coletiva. Tratado de Saúde Coletiva. 2ª edição. Revista Aum, São Paulo, 2012. p 137-170.

3. CECCIM RB. Conexões e fronteiras da interprofissionalidade: forma e formação. Interface, 2018; 22(2): $1739-1749$.

4. CERVO EB, et al. Interprofissionalidade e Saúde Mental: uma revisão integrada. Psicologia e Saúde em Debate, 2020; 6(2): 260-272.

5. COSTA MV, et al. Aspectos institucionais para a adoção da Educação Interprofissional na formação em enfermagem e medicina. Saúde em Debate, 2019; 43(1): 64-76.

6. FARIAS-SANTOS BC, NORO LR. PET-Saúde como indutor da formação profissional para o Sistema Único de Saúde. Ciência e Saúde Coletiva, 2017; 22 (3): 997-1004.

7. FERREIRA VS, et al. PET-Saúde: uma experiência prática de integração ensino-serviço-comunidade. Revista Brasileira de Educação Médica, 2012; 36(1): 147-151.

8. GRANDO MK, DALL'AGNOL CM. Desafios do processo grupal em reuniões de equipe de estratégia de saúde da família. Escola Anna Nery, 2010; 14(3): 504-510.

9. GRZYBOWSKI LS, et al. O que aprendi com o PET? Repercussões da Inserção no SUS para a Formação Profissional. Revista Brasileira de Educação Médica, 2017; 41(4): 505-514.

10. GUETERRES EC, et al. Educación para la salud en el contexto escolar: estudio de revisión integradora. Enfermería Global, 2017,16(2): 464-499.

11. HADDAD AE, et al. Pró-Saúde e PET-Saúde: a construção da política brasileira de reorientação da formação profissional em saúde. Revista Brasileira Educação Médica, 2012; 36 (1): 03-04.

12. MACHADO MH, NETO FR. Gestão da Educação e do Trabalho em Saúde no SUS: trinta anos de avanços e desafios. Revista Ciência e Saúde Coletiva, 2018.

13. MADRUGA LMS, et al. The PET-Family Health and the education of health professionals: students' perspectives. Inteface, 2015; 19(1):805-16.

14. MATUDA CG, et al. Colaboração interprofissional na Estratégia Saúde da Família: implicações para a produção do cuidado e a gestão do trabalho. Ciência e Saúde Coletiva, 2015; 20(8): 2511-2521.

15. MORAIS FR, et al. A importância do PET-Saúde para a formação acadêmica do enfermeiro. Trabalho, Educação e Saúde, 2012; 10(3): 541-551.

16. SILVA AN, et al. Perfil epidemiológico e medidas profiláticas para a influenza na comunidade acadêmica da Universidade de Passo Fundo, Revista Eletrônica Acervo Saúde , 2020, 56(56);e4113

17. PEDUZZI M. O SUS é interprofissional. Interface, 2016; 20(56): 199-201.

18. PREVIATO G, BALDISSERA V. A comunicação na perspectiva dialógica da prática interprofissional colaborativa em saúde na Atenção Primária à Saúde. Interface, 2018; 22(2): 1535-1547.

19. RIBEIRO KG, et al. Educação e saúde em uma região em situação de vulnerabilidade social: avanços e desafios para as políticas públicas. Revista Interface- Comunicaçao, Saúde, Educação, 2018;22(1):1387-1398.

20. SOUSA FM, et al. Educação interprofissional e educação permanente em saúde como estratégia para a construção de cuidado integral na Rede de Atenção Psicossocial. Revista de Saúde Coletiva, 2020; 30(1): 1-21.

21. VIACAVA F, et al. SUS: oferta, acesso e utilização de serviços de saúde nos últimos 30 anos, Revista SUS: oferta, acesso e utilização de serviços de saúde nos últimos 30 anos. Ciência e Saúde Coletiva, 2018 ;23(6):1751-1762. 